\title{
Self-adaptive Video Target Tracking and Application based on Particle Filter
}

\author{
Fankun MENG ${ }^{1, a}$, Hao TIAN $^{2, b}$ \\ ${ }^{1}$ School of Electronic and Control Engineering, Chang'an University, Xi'an City, Shannxi, 710064, \\ China \\ ${ }^{2}$ School of Electronic and Control Engineering, Chang'an University, Xi'an City, Shannxi, 710064, \\ China
}

Keywords: Simulation; Accuracy; Differential Evolution; Filtering Congestion-Degree; Tracking

\begin{abstract}
In order to promote tracking and positioning accuracy of wireless sensor network node and reduce energy consumption further, a multi-objective congestion-degree differential optimization and Bayes quantification variation filtering and estimating WSN tracking and positioning algorithm is presented in the Thesis. First of all, for positioning problem, Bayes quantification variation filtering method is adopted to estimate the next position area of the objective and quantification variation filtering method is used to select the proper positioning parameter and node and multi-objective parameter optimization model of quantification variation filtering is also designed. Secondly, as optimization accuracy of traditional multi-objective optimization algorithm is not high, multi-objective differential evolution algorithm based on individual congestion of certain population is designed so as to optimize parameters of quantification variation filtering algorithm and realize multi-objective optimization of filtering parameter. At last, experiment simulation shows that, the algorithm can realize tracking and positioning of targeted node effectively and can also save energy consumption.
\end{abstract}

\section{Introduction}

There are many definitions of wireless sensor network (WSN for short). In practical application, it can be deemed as a highly districted network structure constituted by mutual communication of large quantity of small-volume and light-weight sensors. It can be used to detect environment or physical parameters of system such as temperature and pressure. In WSN network, network node is powered by storage battery. The battery can not be charged or replaced and it is the main component influencing node even life of the network. Therefore, energy consumption is the key factor which needs to be taken into consideration during design of $\mathrm{WSN}^{[1]}$.

\section{Multi-Objective Differential Evolution Algorithm}

\section{Non-dominated sorting}

Initial population shall be sorted based on non-dominated relationship and literature [6] quick sort method shall be adopted:

Step1: the following items shall be implemented for each individual $P$ in population $P$ :

(1) Suppose $S_{p}=\phi, n_{p}=0 . \quad p$ is the individual in main population $P . S_{p}$ is used to store individual dominated by $p$ in the main population. $n_{p}$ is the quantity of individual dominating $p$;

(2) For each individual $q$ in $P$, if $p \succ q, S_{p}=S_{p} \cup\{q\}$; otherwise $q \succ p, n_{p}=n_{p}+1$;

(3) If $n_{p}=0$, rank of individual $p \quad p_{\text {rank }}=1 . p$ shall be added into current Pareto front, namely $F 1=F 1 \cup\{p\}$.

Step2: the following operations shall be implemented until it meets condition $F_{i}=\phi$ :

(1) Suppose $Q=\phi$ and it is used to store $F_{i}$ temporarily;

(2) For each individual $p$ in $F_{i}$, the following operations shall be implemented: for each 
individual $q$ in $S_{p}$, the following operations shall be implemented: Suppose $n_{q}=n_{q}-1$, if $n_{q}=0, q$ is only dominated by $p$. Rank of $q$ shall be set $q_{\text {rank }}=i+1$ and $Q=Q \cup q$;

(3) Suppose $i=i+1$;

(4) Suppose $F_{i}=Q$ and (2-n)th front $F_{2} \sim F_{n}$ will be gained successively.

As two parameters are added in sorting process of the algorithm: $S_{p}$ and $n_{p}$ and population diversity is controlled artificially, the algorithm is superior to the sorting method in literature [2] theoretically.

\section{Calculation of congestion}

In evolutionary process of differential population, individual with high adaptability and relatively small density distance away from other individuals shall be reserved. Set there are $r$ sub-objectives $f_{1}, f_{2} \ldots f_{r}$ and congestion of individual $i$ is $P[i]_{d i s} . P[i] . m$ is the function value of individual $i$ on sub-objective $m$.Calculation method of congestion:

$$
P[i]_{d i s}=\sum_{k=1}^{r}\left(P[i+1] \cdot f_{k}-P[i-1] \cdot f_{k}\right)
$$

If population scale is $N$, time complexity for congestion sorting of $r$ sub-objectives under the worst condition is $O(r N \log N)$. Time complexity for calculation of congestion is $O(r N)$. Therefore, time complexity of the algorithm is $O(r N \log N)$.

\section{Multi-objective differential evolution algorithm steps}

Step1: set population scale $N$, maximum iteration number gen, top and bottom limitation of population $X$ Vmax and $X V \min$. Population pop shall be initialized based on methods specified in Section 3.1 and evaluation, non-dominated sorting and congestion calculation shall be conducted. Suppose $i=1$;

Step2: based on binary championship methods, $N / 2$ individuals shall be selected from pop to form parent individual parent_pop. Parent population shall undergo differential operation (variation and interlace operation) and population pop1will be generated and its scale is $N / 2$;

Step3: pop1 and pop shall be combined to generate intermediate_pop and its individual shall undergo non-dominated sorting and congestion calculation. $N$ individuals shall be selected according to sorting grade and degree of congestion to form a new population pop;

Step4: $i=i+1$, if $i \leq$ gen , back to Step2; if $i>$ gen , the step will go on;

Step5: output pop, namely pareto optimum solution provided by multi-objective differential evolution algorithm.

\section{Simulation Experiment and Analysis}

Test function adopted is:

$$
\text { MOP1: }\left\{\begin{array}{l}
f_{1}(x)=1-e^{-4 x_{1}} \sin ^{6}\left(6 \pi x_{1}\right) \\
f_{2}(x)=g(x)\left(1-\left(f_{1}(x) / g(x)\right)^{2}\right) \\
g(x)=1+9\left(\sum_{i=1}^{6} x_{i} / 4\right)^{0.25}
\end{array}\right.
$$

Where, value range of $x$ is $0 \leq x_{i} \leq 1, i=1, \ldots, 6$.

$$
\text { MOP2: }\left\{\begin{array}{l}
f_{1}(x)=x_{1} \\
f_{2}(x)=g \times\left(1-\sqrt{f_{1} / g}\right) \\
g=1+9 \times \sum_{i=2}^{n} x_{i} /(n-1)
\end{array}\right.
$$

Where, value range of $x$ is $0 \leq x_{i} \leq 1, i=1, \ldots, 12$.

Parameter shall be set. Population scale $N=200$, evolution algebra gen $=500$, NSGA2 II crossover and mutation operators are 20, CNMDE crossover probability factor $C R=0.3$, zoom factor $F=0.6$. Comparison algorithm shall be NSGA II ${ }^{[13]}$, SOEA, SPEA and VEGA. Comparison results of Pareto optimum solution solved by five algorithms are shown in Fig.1. 
Fig.1 (a)(b) are schematic diagrams for comparison of Pareto optimum solution of two test functions respectively. As above sensor network quantification variation filtering and parameter optimization are two-objective optimization problems, when multi-objective optimization algorithm is tested, two two-objective standard test functions shall be selected for simulation comparison. When performance of multi-objective optimization algorithm is evaluated, two indexes will be used: leading edge and uniformity of solution. Fig.1 shows that, for leading edge of the solution, CNMDE algorithm in the Thesis is better than comparison algorithm obviously. For uniformity of solution, CNMDE algorithm is superior to VEGA and SOEA algorithms obviously and slightly superior to NSGA II and SPEA algorithms. Fig.2 is the box diagram of Pareto optimum solution sets from five algorithms and Fig. 2 shows that, CNMDE algorithm is better than the contrasted algorithm in terms of accuracy and distribution of the solution. Above contrast experiments verify validity of the algorithm in standard test function and wireless sensor network positioning algorithm of the algorithm will be researched in the following parts.

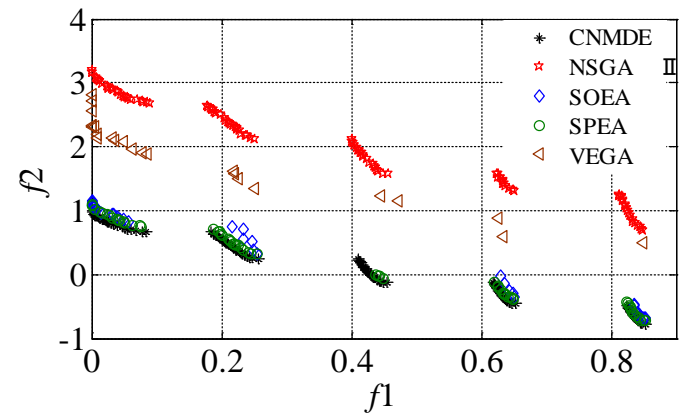

(a) MOP1 optimization results

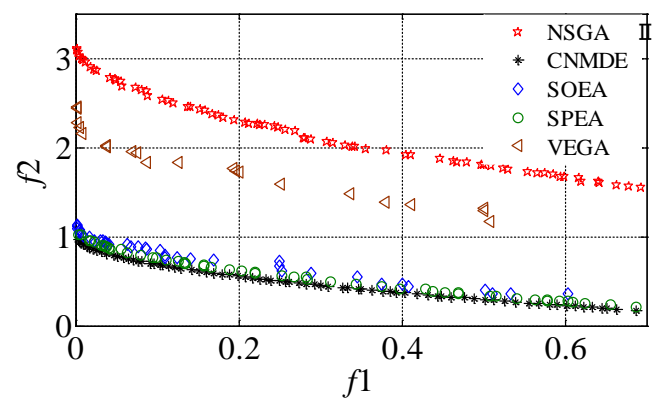

(a) MOP2 Optimization Results

Fig.1. Schematic diagram for comparison of optimum solution set

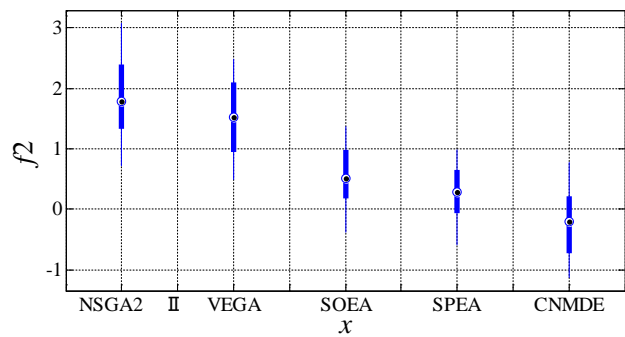

(a) Solution distribution of MOP1

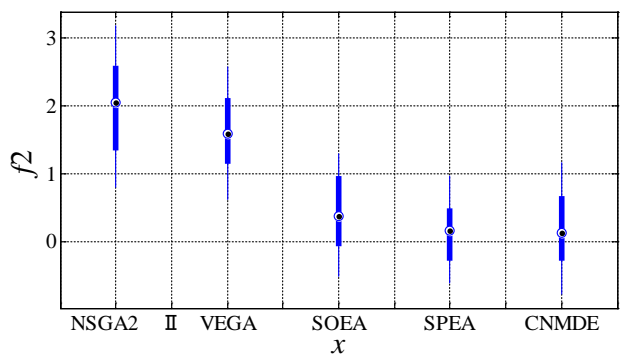

(b) Solution distribution of MOP2

Fig.2. Box Diagram of pareto optimum solution set 


\section{Conclusion}

In order to promote tracking and positioning accuracy of wireless sensor network node and reduce energy consumption, a multi-objective congestion-degree differential optimization and Bayes quantification variation filtering and estimating WSN tracking and positioning algorithm is presented in the Thesis. Improved multi-objective differential evolution algorithm realizes effective optimization for multi-objective parameter model of quantification variation filtering. In this way, energy consumption of positioning is saved and positioning accuracy is also promoted. Simulation results verify validity of the algorithm.

\section{Acknowledgement}

The Natural Science Foundation of China under Grant No.61473047.

\section{Reference}

[1] Lv Z, Tek A, Da Silva F, et al. Game on, science-how video game technology may help biologists tackle visualization challenges[J]. PloS one, 2013, 8(3): e57990.

[2] Jiang D, Ying X, Han Y, et al. Collaborative multi-hop routing in cognitive wireless networks[J]. Wireless Personal Communications, 2016, 86(2): 901-923.

[3] Lin Y, Yang J, Lv Z, et al. A self-assessment stereo capture model applicable to the internet of things[J]. Sensors, 2015, 15(8): 20925-20944.

[4] Lv Z, Halawani A, Feng S, et al. Multimodal hand and foot gesture interaction for handheld devices[J]. ACM Transactions on Multimedia Computing, Communications, and Applications (TOMM), 2014, 11(1s): 10. 